\title{
Crossmodal action: modality matters
}

\author{
Lynn Huestegge $\cdot$ Eliot Hazeltine
}

Published online: 10 September 2011

(c) Springer-Verlag 2011

Research on multitasking harkens back to the beginnings of cognitive psychology. The central question has always been how we manage to perform multiple actions at the same time. Here, we highlight the role of specific inputand output-modalities involved in coordinating multiple action demands (i.e., crossmodal action). For a long time, modality- and content-blind models of multitasking have dominated theory, but a variety of recent findings indicate that modalities and content substantially determine performance. Typically, the term "input modality" refers to sensory channels (e.g., visual input is treated differently from auditory input), and the term "output modality" is closely associated with effector systems (e.g., hand vs. foot movements). However, this definition may be too narrow. The term "input modality" sometimes refers to a dimension within a sensory channel (e.g., shape/color in vision). Furthermore, the linkage between output-modalities and effector systems may not be specific enough to illuminate some notorious twilight zones (e.g., to distinguish between hand and wrist movements). As a consequence, we will use "modality" as an umbrella term here to capture various sources of stimulus variability used to differentiate the task-relevant information and sources of motor variability used to differentiate responses.

Many of the pioneering studies involved the observation of dual-task performance in two continuous tasks that typically consisted of complex action sequences (e.g., reading and writing, see Solomons \& Stein, 1896; Spelke,

L. Huestegge $(\bowtie)$

RWTH Aachen University, Aachen, Germany

e-mail: lynn.huestegge@psych.rwth-aachen.de

E. Hazeltine

University of Iowa, Iowa City, USA
Hirst, \& Neisser, 1976). However, it soon became apparent that tighter experimental control was necessary to pinpoint the specific cognitive mechanisms supporting multitasking.

The PRP paradigm: an experimental breakthrough. The development of the psychological refractory period (PRP) paradigm (Telford, 1931; Welford, 1952) provided a methodological breakthrough that allowed researchers to exactly control the flow of information in both tasks. The PRP paradigm involves two elementary tasks with a limited set of clearly defined stimuli and responses. The mechanisms underlying multitasking are studied by systematically manipulating the temporal overlap of the two tasks, which is achieved by varying the delay between the presentations of the stimuli for the two tasks (stimulus onset asynchrony, SOA). The PRP effect refers to the typical finding that reaction times (RTs) for the second task increase with decreasing SOA, an effect that has been replicated in numerous studies with a variety of stimulus and response modalities (see Bertelson, 1966; Pashler, 1994; Smith, 1967).

The RSB model: a powerful explanatory concept? The most influential and elegant account of the PRP effect has been the response selection bottleneck (RSB) model (Telford, 1931; Welford, 1952). A starting assumption of the RSB model is that tasks at hand can be divided into three successive cognitive processing steps, namely perceptual processing (i.e., stimulus encoding/categorization), response selection (i.e., deciding which response corresponds to the stimulus according to the task rules), and response execution processes. In a number of experiments, the duration of each of these processing stages was systematically manipulated for each of the two tasks (see Pashler, 1994). As a result, the most convincing hypothesis to accommodate the corresponding findings was the assumption that perceptual processing and response 
execution can principally proceed in parallel with any processing stage of the other task, whereas processing associated with response selection could only be performed for one task at a time (serial processing). The RSB model dominated dual-task research in the $80 \mathrm{~s}$ and $90 \mathrm{~s}$, because it was a simple and straightforward account that could readily explain a great variety of effects in dual-task research, especially within the context of the PRP paradigm (Pashler, 1994).

It should be noted that from a historical point of view, the RSB model was strongly inspired by fundamental assumptions from early cognitive psychology, which heavily borrowed explanatory concepts from Information Theory. According to Information Theory (Shannon, 1948), information transmission is conceptualized as a content-blind process of transmitting coded information (i.e., binary units) between a sender and a receiver. Applied to human beings, this theory holds that people are basically information processing systems (Broadbent, 1958; Neisser, 1967). As a consequence, the computer served as a central theoretical metaphor for explaining psychological phenomena. The concept of a content-blind, unitary, and a-modal central processing unit (CPU) obviously resonates with the RSB model, both in the assumption of processing steps within each task that are unspecific to modalities and content, and in the assumption of a unitary, central, amodal and content-blind response selection processor (Lachman, Butterfield, \& Lachman, 1979).

Strategies and modalities in dual-tasks In recent years, the RSB model has been attacked on at least two fronts: first, it has been argued that serial central processing might not be a generic property of our cognitive system, but rather a strategic response to the (somewhat artificial) serial stimulus presentation in the PRP paradigm. Second, converging evidence has suggested that response selection is not a content- and modality-blind unitary entity, but rather strongly dependent upon the specific input- and outputmodalities involved.

Evidence for the first claim comes from Schumacher et al. (2001) and, more recently, from Israel and Cohen (2011). Schumacher et al. (2001) had participants perform an auditory-vocal (AV) task and a visual-manual (VM) task, which-after some practice-were performed without dual-task costs. However, in a condition in which the stimuli of the AV and the VM task were presented with a variable SOA, dual-task costs (i.e., a PRP effect) reemerged. Thus, even though participants were able to perform both tasks without costs, the introduction of the SOA manipulation appeared to induce a substantial change in cognitive strategy. Similar effects are reported by Israel and Cohen (2011). Taken together, these results suggest that the PRP paradigm may not be a valid model to test to what extent two tasks can principally be processed simultaneously, because its inherent structure (i.e., the variable SOA) seems to impose a task-specific serial processing strategy. This severely limits the validity of the PRP paradigm as a general model for cognitive processes during multitasking.

Evidence for the second claim, i.e. the modality-dependence of response selection, was originally provided by Greenwald (1972) and Greenwald and Shulman (1973). They reasoned that the extent to which a stimulus resembles normally occurring sensory feedback of the response (e.g., saying a word in response to hearing it) affects the amount of dual-task costs. This assumption also included the hypothesis that some input-modalities should go well with specific output modalities (e.g., auditory input- and vocal output), but was more specific in that it additionally required identity between the stimulus and the sensory feedback of the response (e.g., saying " $A$ " in response to hearing " $A$ "). More general evidence for preferred input-output modality combinations comes from a number of more recent studies (e.g., Hazeltine \& Ruthruff, 2006; Hazeltin, Ruthruff \& Remington, (2006); Levy \& Pashler, 2001; Stelzel, Schumacher, Schubert, \& D'Esposito, 2006). By manipulating the specific pairings of input- and output-modalities in dual-tasks, these studies demonstrated that some tasks, specifically AV and VM tasks, can be performed without significant costs after practice, while other task combinations (e.g., AM and VV tasks) show persistent dual-task costs even after substantial practice. These observations appear to be at variance with core assumptions of a simple content-blind, modality-independent RSB model, according to which there should be no reason to assume that the specific pairings of input- and output-modalities should substantially affect the performance. As a result, many researchers largely abandoned the idea of content-blind, modality-independent central cognitive processing. Instead, it seemed as if modalities matter in dual-task control, although the specific mechanisms behind the reported effects remained elusive (but see Hazeltine \& Wifall, 2011; Schumacher, Schwarb, Lightman, \& Hazeltine, 2011).

Resource theory: a way out? However, dual-task research has not always been dominated by a content-blind theoretical approach. While early resource accounts were based on the assumption of a central, unitary resource for all cognitive activities (e.g., Kahneman, 1973), the most prominent competitor for the RSB model has been multiple resource theory, which assumed that dual-task costs arise from competition for access to domain-specific resources (Navon \& Gopher, 1979; Wickens, 1980, 2002). For example, Wickens, Sandry, and Vidulich (1983) proposed a model of dual-task processing in which competition could emerge at any of the three processing stages. Furthermore, they assumed that some combinations of stimuli, central codes, and responses were more resistant to dual- 
task interference than other combinations. Thus, the idea of multiple resources represented a viable theoretical alternative to the content-blind RSB model (see also Wickens \& Liu, 1988).

Unfortunately, some general shortcomings of resource theory prevented greater success. The term "resource" was mainly defined with respect to the observation of dual-task costs, so that its concept appeared to be circular: whenever two tasks produced dual-task costs, shared resources were assumed, whereas the absence of dual-task costs was interpreted in terms of separate resources (see Allport, 1980; Navon, 1984). As a result, many researchers abandoned resource theory; although some of its intuitions still appear to be helpful in further explaining the sources of dual-task costs (see Hazeltine \& Wifall, 2011).

An alternative approach which avoided the pitfalls of resource theory was the notion of crosstalk. This term was introduced by Navon (1984) and Navon and Miller (1987) and successfully challenged RSB theory. The basic idea was that the outcome of the processing for one task interferes with that of another task. In line with this assumption, Navon and Miller reported data suggesting that the relatedness of two tasks substantially affected dual-task costs, a finding that further supported content-based accounts of multitasking.

Set-level compatibility: an explanation for modality effects? It is also possible to think of the effects of modalitypairings in terms of set-level compatibility (see Fitts \& Deininger, 1954; Kornblum, Hasbroucq, \& Oman, 1990). Set-level compatibility refers to the benefits (or costs) in performance associated with the mapping of one set of stimuli to one set of responses, regardless of the specific mappings of individual stimuli to individual responses (i.e., element-level compatibility). According to this framework, dual-task costs are reduced because certain pairings are more compatible than others. However, this explanation is also ultimately unsatisfactory: in many cases, the effects of modality pairings are only seen in dual-task conditions; single-task RTs do not appear affected, making it difficult to apply this concept to modality-pairing phenomena without adding many counter-intuitive assumptions.

Modality neglect in dual-task research As noted above, previous studies have convincingly shown that the specific pairings of stimulus and response modalities involved in a dual-task setting are important to consider. Thus, it is remarkable that most dual-task research uses only two input- and output modality-pairings (i.e., visual/auditory input, and manual/vocal output). Most of the early PRP studies involved manual responses in both tasks (sometimes even with the same finger, which effectively ruled out simultaneous responses in the first place; see Pashler, 1994, for a review). In the following, manual responses were combined with vocal responses (Pashler, 1990) or foot responses (Osman \& Moore, 1993). There were also few studies combining vocal and foot responses (Pashler \& Christian, 1994, as cited in Pashler, 1994). While these combinations provided further evidence for a robust PRP effect, an attempt to demonstrate a PRP effect for the combination of manual and basic saccade responses failed (Pashler, Carrier, \& Hoffman, 1993).

To back up the claim that dual-task studies in the literature still primarily focus on only two input- and outputmodalities (i.e., visual/auditory input- and manual/vocal output), we conducted a meta-analysis (using the "web of knowledge" database) of all studies from the past 5 years that refer to the term "dual task" in their title $(N=188)$. Based on the abstracts, we determined the input- and output modalities involved in each study. In 87 cases, the abstract did not contain sufficient information. Another 48 studies referred to the effects of additional task demands on postural control, which was regarded as a special case of dualtask control and therefore excluded from further analysis.

Figure 1 depicts the frequency of specific modality combinations in the remaining 53 studies. As the figure suggests, nearly all dual-task studies concentrate on visual or auditory input- and manual- or vocal output. Only one study utilized tactile stimulation, and only a very limited number of studies involved alternative response modalities, such as saccadic or pedal responses.

With respect to the growing interest in input-output compatibility issues, it appears especially interesting that task combinations, where a common body part is involved in input- and output related processing, are completely neglected, e.g. tactile-manual tasks, or visual-oculomotor tasks. It is also evident that some output modalities are completely missing, e.g., facial expressions, gesturing (which often involves more than just manual movements), eye blinks, respiratory responses etc.

Crossmodal action Given that recent research has highlighted the role of modalities in dual-task research and that most dual-task data and theory have been based on a limited array of input- and output-modalities, it appears imperative to focus much more on the role of modalities in dual-task control. Thus, we would like to coin the term "Crossmodal Action" for underlining the important role of modalities and how they are linked through S-R pairings in multitasking. Originally, this term was introduced by $\mathrm{Hu}-$ estegge and Koch (2010) to highlight the important role of modalities in multitasking on the output side of information processing by utilizing experimental paradigms allowing for perfect control over stimulus-related processing (see also Huestegge \& Koch, 2009). Here, however, we advocate for a broader definition of the term "Crossmodal Action", which more generally refers to a research program that highlights the role of content and modalities on the input and output side of multitasking. 
Fig. 1 Modalities involved in a representative sample of dualtask studies from the past 5 years. The $y$-axis refers to the number of studies, while the $x$ axis refers to the specific modality combinations. The first pair of letters represents inputand output modalities of one task, whereas the second pair of letters represents input- and output modalities of the second task. Input modalities: $V$ visual, $A$ auditory, $T$ tactile. Output modalities: $M$ manual, $V$ vocal, $S$ saccadic, $P$ pedal

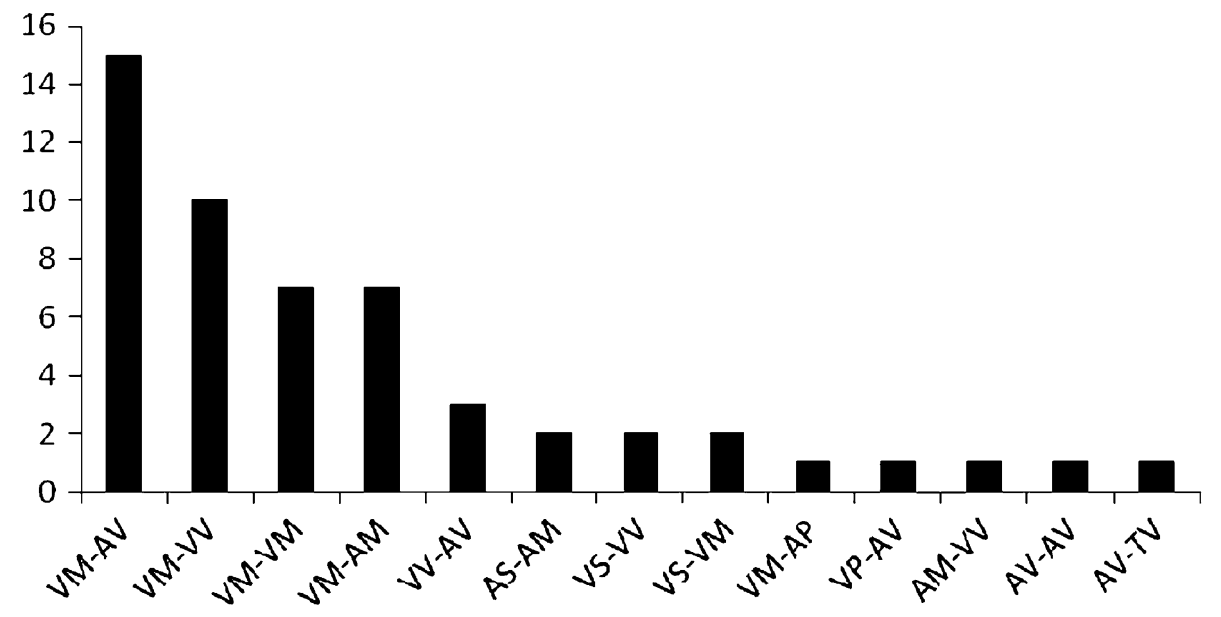

Task combinations (input-/output-modalities)
The special issue on crossmodal action In the current Special Issue, we focus on the question of how crossmodal processing influences the control of action. The range of specific research questions is broad: how are crossmodal actions represented in the cognitive system and in the brain?, how is information integrated across different input- and output-modalities?, what is the time course of the integration of modality specific processing?, do the modality pairings determine whether the central processing is serial or parallel?, do specific pairings of inputs and outputs engage specialized processes?, in what way do crossmodal action demands influence performance in applied settings, e.g. during navigation in traffic? these questions touch on fields as diverse as memory, attention, and action planning. Furthermore, we suspect that a wide range of empirical methods may well provide a useful insight into any one of these research questions. It is this breadth of approaches toward a single theme that we hope to capture here. While the emphasis of many studies is on the role of modalities in terms of sensory channels and effector systems, this does in no way imply that other content-related aspects play a subordinate role, for example, effects of specific processing content (see Atchley, Dressel, Jones, Burson, \& Marshall, 2011), or of content dimensions (e.g., color vs. shape processing, see the related concept of "modules" discussed in Israel \& Cohen, 2011).

Overview of contributions In the following, we present a brief overview of the contributions. These can be roughly characterized based on their theoretical perspective and the underlying methodology.

The paper by Hazeltine \& Wifall (2011) digs deeper into the potential mechanisms underlying the effects of input-output modality compatibility. As a theoretical background, they primarily refer to domain-specific accounts of dual-task control (e.g., multiple resource frameworks and/or crosstalk models). More specifically, they explore to what extent response selection processes may engage domain-specific working memory processes, which in turn may account for input-output modality pairing effects. In their paradigm, they combined a choice RT task (with variable response modalities involved) with a simultaneous working memory task (with variable domains involved). While vocal responses interfered with auditory working memory, manual responses interfered with visuospatial working memory, suggesting that response selection engages domain-specific memory processes. This can be interpreted as further evidence against the assumption of a unitary, supra-modal response selection mechanism.

The study by Stelzel and Schubert (2011) further analyzes the conditions under which input- and outputmodality pairing effects occur within the PRP paradigm. More specifically, they focused on the potential effects of S-R compatibility and crosstalk. Their data indicated that modality pairing effects are also present in task situations where S-R mappings are compatible. However, additional sources of dual-task interference in a modality compatible dual task could overwrite the pronounced PRP effect previously shown for modality incompatible tasks. Taken together, the study shows how modality pairings interact with other sources of interference in dual-task situations.

The study by Stephan and Koch (2011) takes a closer look at input-output modality compatibility within a task switching paradigm by examining the role of dimensional overlap between the two tasks based on spatially compatible S-R mappings in both tasks. Interestingly, the inputoutput modality compatibility effect remained intact even when the two tasks did not involve dimensional overlap, suggesting that input-output modality mappings affect the response selection by influencing between-task crosstalk not on the level of specific response codes, but rather on the level of modality-specific processing pathways. 
The study by Schumacher et al. (2011) takes a similar theoretical viewpoint as the Hazeltine and Wifall paper, but rather focuses on the effects of different input-modalities on brain activation during response selection. More specifically, they utilized a variable that is known to affect the response selection processes (i.e., flanker congruency) and studied its effects on brain activation for tasks involving visual versus auditory stimuli. The results suggested that brain activation varied as a function of input-modalities, despite the fact that stimuli and tasks were conceptually identical. Again, this finding appears to be compatible with the assumption that dual-task processing strongly depends on the specific modalities involved.

The study by Israel and Cohen (2011) further undermines the assumption that the PRP paradigm (and the corresponding RSB framework) is a valid, universal model of dual-task performance in general. More specifically, they trained participants to perform two tasks simultaneously without dual-task costs in conditions without a temporal separation between the stimuli for both tasks ( $\mathrm{SOA}=0 \mathrm{~ms}$, see also Schumacher et al., 2001).

Interestingly, whenever this condition was mixed with other conditions involving sequential stimulus presentation (SOA $>0 \mathrm{~ms}$ ) within a block of trials, a PRP effect emerged. Obviously, the mere presence of trials involving sequential stimulus presentation within the same block automatically induced a costly, serial cognitive processing strategy in participants (see also Meyer \& Kieras, 1997), even though they were (principally) perfectly able to perform both tasks without costs. These results also suggest that the external validity of previous PRP studies may be more limited than previously assumed.

Apart from these specific results, the paper also briefly describes an alternative to the view that modalities and their specific combinations are an important source of dualtask costs. More specifically, the authors refer to their Dimension-Action model of dual-task control, according to which the visual system is segregated into separate modules such as color, shape, and word. Importantly, they claim that dual-task costs should arise whenever two tasks involve processing within the same modules (see Magen \& Cohen 2007). This framework is in line with modalitybased accounts of dual-task control in that it also refers to interference based on the specific content of the tasks, but obviously focuses on different key aspects (i.e., modules instead of modalities).

The study by Atchley et al. (2011) adds an applied perspective to the present research topic. They demonstrate that it is particularly difficult to be engaged in a (visuospatial) driving task while at the same time performing a spatial (vs. non-spatial) verbal task. Moreover, a second experiment suggested that this effect might relate to shared right parietal resources. Unlike in many other studies reported in this Special Issue, the critical comparison here is between two task demands which share exactly the same modalities. Thus, the critical source of dual-task costs here seems to be related to code interference, i.e. to the fact that two tasks draw on common limited visuo-spatial processing resources. However, it seems important to note that in this particular study there was no evidence of within-trial spatial interference, for example, when the two tasks in one particular trial involved processing of a different (vs. the same) spatial code.

The review paper by Huestegge (2011) presents evidence for the claim that multitasking research may benefit from utilizing a greater variety of modalities. More specifically, the paper presents an overview of the role of saccades in multitasking. While for a long time, eye movements were mainly regarded as a means to measure attention, recent research strongly suggests that they both exhibit and cause dual-task costs in the context of other actions, and should therefore also be regarded as an ordinary response modality. The paper demonstrates that most of the evidence regarding saccades in multitasking points to parallel central processing, and corresponding dual-task costs appear to be mainly associated with crosstalk based on within- and/or between-trial interference.

Finally, the review paper by Wylie, Sumowski, and Murray (2011) reports studies that are centered around the question of whether the assumption of cognitive control processes is necessary to explain the behavior in dual-task situations. They review the evidence for and against this assumption in both the behavioral and the neuroscience domain. Specifically, they focus on the recent empirical work on task switching studies in which participants switch between tasks involving different input-modalities (i.e., visual vs. auditory). They discuss to what extent these studies may represent a step forward in the study of the nature of cognitive control.

In summary, the present studies point to several potential sources of dual-task costs, which do not appear to be mutually exclusive. These include domain-specific working memory interference (Hazeltine \& Wifall, 2011), strategic effects based on task context (Israel \& Cohen, 2011), modality-specific processing pathways (Schumacher et al., 2011; Stephan \& Koch, 2011), interference based on common (spatial) codes (Atchley et al., 2011) or on shared visual modules (shape, color, word, see Israel \& Cohen, 2011), and RSB models (at least for results within the PRP paradigm, see Israel \& Cohen, 2011; Stelzel \& Schubert, 2011). Further papers additionally discuss the role of cognitive control processes (Wylie et al., 2011), and within- and between-trial crosstalk (Huestegge, 2011).

Taken together, the findings present a complex array of potential sources of dual-task costs that is certainly not as 
elegant as the assumption of a single theoretical entity (e.g., resource, bottleneck). However, it may come much closer to reflect the actual complexity of the human mind and brain. The aim is to lay the groundwork for an overarching the theoretical framework by describing the range of potentially related phenomena that stem from the pairings of stimulus and response modalities.

\section{References}

Allport, D. A. (1980). Attention and performance. In G. L. Claxton (Ed.), Cognitive psychology: New directions (pp. 112-153). London: Routledge.

Atchley, P., Dressel, J., Jones, T., Burson, R., \& Marshall, D. (2011). Talking and driving: applications of crossmodal action reveal a special role for spatial language. Psychological Research. doi: 10.1007/s00426-011-0342-7 (this volume)

Bertelson, P. (1966). Central intermittency twenty years later. The Quarterly Journal of Experimental Psychology, 18, 153-163.

Broadbent, D. E. (1958). Perception and Communication. London: Pergamon.

Fitts, P. M., \& Deininger, R. L. (1954). S-R compatibility: correspondence among paired elements within stimulus and response codes. Journal of Experimental Psychology, 48, 483-492.

Greenwald, A. G. (1972). On doing two things at once: timesharing as a function of ideomotor compatibility. Journal of Experimental Psychology, 94, 52-57.

Greenwald, A. G., \& Shulman, H. G. (1973). On doing two things at once: II. Elimination of the psychological refractory period effect. Journal of Experimental Psychology, 101, 70-76.

Hazeltine, E., \& Ruthruff, E. (2006). Modality pairing effects and the response selection bottleneck. Psychological Research, 70, 504-513.

Hazeltine, E., \& Wifall, T. (2011). Searching working memory for the source of dual-task costs. Psychological Research. doi: 10.1007/s00426-011-0343-6 (this volume)

Hazeltine, E., Ruthruff, E., \& Remington, R. W. (2006). The role of input and output modality pairings in dual-task performance: Evidence for content-dependent central interference. Cognitive Psychology, 52, 291-345.

Huestegge, L. (2011). The role of saccades in multitasking: towards an output-related view of eye movements. Psychological Research. doi:10.1007/s00426-011-0352-5 (this volume)

Huestegge, L., \& Koch, I. (2009). Crosstalk between simultaneously executed saccades and manual responses. Journal of Experimental Psychology: Human Perception and Performance, 35, 352-362.

Huestegge, L., \& Koch, I. (2010). Crossmodal action selection: Evidence from dual-task compatibility. Memory and Cognition, $38,493-501$.

Israel, M., \& Cohen, A. (2011). Involuntary strategy-dependent dual task performance. Psychological Research. doi:10.1007/s00426011-0359-y (this volume)

Kahneman, D. (1973). Attention and effort. Englewood Cliffs, NJ: Prentice-Hall

Kornblum, S., Hasbroucq, T., \& Osman, A. (1990). Dimensional overlap: Cognitive basis of stimulus-response compatibility-A model and taxonomy. Psychological Review, 97, 253-270.

Lachman, R., Butterfield, E., \& Lachman, J. L. (1979). Cognitive psychology and information processing. Mahwah: Erlbaum.
Levy, J., \& Pashler, H. (2001). Is dual-task slowing instruction dependent? Journal of Experimental Psychology: Human Perception and Performance, 27, 862-869.

Meyer, D. E., \& Kieras, D. E. (1997). A computational theory of executive cognitive processes and multiple-task performance: Part 1 Basic mechanisms. Psychological Review, 104, 3-65.

Navon, D. (1984). Resources-A theoretical soupstone? Psychological Review, 91, 216-234.

Navon, D. (1985). Attention division or attention sharing. In M. I. Posner \& O. S. M. Marin (Eds.), Attention and performance XI (pp. 133-146). Hillsdale: Erlbaum.

Navon, D., \& Gopher, D. (1979). On the economy of the human information processing system. Psychological Review, 86, 214-255.

Navon, D., \& Miller, J. (1987). Role of outcome conflict in dual-task interference. Journal of Experimental Psychology: Human Perception and Performance, 13, 435-448.

Neisser, U. (1967). Cognitive psychology. New York: AppletonCentury-Crofts.

Osman, A., \& Moore, C. (1993). The locus of dual-task interference: Psychological refractory effects on motor-related brain potentials. Journal of Experimental Psychology: Human Perception and Performance, 19, 1292-1312.

Pashler, H. (1990). Do response modality effects support multiprocessor models of divided attention. Journal of Experimental Psychology: Human Perception and Performance, 16, 826-842.

Pashler, H. (1994). Dual-task interference in simple tasks: Data and theory. Psychological Bulletin, 116, 220-244.

Pashler, H., Carrier, M., \& Hoffman, J. (1993). Saccadic eye movements and dual-task interference. Quarterly Journal of Experimental Psychology, 46A, 51-82.

Schumacher, E. H., Seymour, T. L., Glass, J. M., Fencsik, D. E., Lauber, E. J., Kieras, D. E., et al. (2001). Virtually perfect time sharing in dual-task performance: uncorking the central cognitive bottleneck. Psychological Science, 12, 101-108.

Schumacher, E., Schwarb, H., Lightman, E., \& Hazeltine, E. (2011). Investigating the modality specificity of response selection using a temporal flanker task. Psychological Research (this volume)

Shannon, C. E. (1948). A mathematical theory of communication. Bell System Technical Journal, 27, 379-423. 623-656.

Smith, M. C. (1967). Theories of the psychological refractory period. Psychological Bulletin, 67, 202-213.

Solomons, L. M., \& Stein, G. (1896). Normal motor automatism. Psychological Review, 3, 492-512.

Spelke, E. S., Hirst, W., \& Neisser, U. (1976). Skills of divided attention. Cognition, 4, 215-230.

Stelzel, C., \& Schubert, T. (2011). Interference effects of stimulusresponse modality pairings in dual tasks and their robustness. Psychological Research. doi:10.1007/s00426-011-0368-x (this volume)

Stelzel, C., Schumacher, E., Schubert, T., \& D’Esposito, M. (2006). The neural effect of stimulus-response modality compatibility on dual-task performance: An fMRI study. Psychological Research, $70,514-525$.

Stephan, D. N., \& Koch, I. (2011). The role of input-output modality compatibility in task switching. Psychological Research. doi: 10.1007/s00426-011-0353-4 (this volume)

Telford, C. W. (1931). The refractory phase of voluntary and associative responses. Journal of Experimental Psychology, 14, $1-36$.

Welford, A. T. (1952). The "psychological refractory period" and the timing of high-speed performance-A review and a theory. British Journal of Psychology, 43, 2-19.

Wickens, C. D. (1980). The structure of attentional resources. In R. Nickerson (Ed.), Attention and performance VIII (pp. 239-257). Hillsdale: Erlbaum. 
Wickens, C. D. (2002). Multiple resources and performance prediction. Theoretical Issues in Ergonomics Science, 3, 159-177.

Wickens, C. D., \& Liu, Y. (1988). Codes and modalities in multiple resources: A success and a qualification. Human Factors, 30, 599-616.

Wickens, C. D., Sandry, D. L., \& Vidulich, M. (1983). Compatibility and resource competition between modalities of input, central processing and output. Human Factors, 25, 227-248.
Wylie, G., Sumowski, J. F., \& Murray, M. (2011). Are there control processes, and (if so) can they be studied? Psychological Research. doi:10.1007/s00426-011-0354-3 (this volume) 